\title{
Protein interaction and microRNA network analysis in osteoarthritis meniscal cells
}

\author{
M. Wang, C. Liu, Y. Zhang, Y. Hao, X. Zhang and Y.M. Zhang \\ Department of Orthopedics, Xinqiao Hospital, \\ Third Military Medical University, Chongqing, China \\ Corresponding author: C. Liu \\ E-mail: 52solar@sina.cn
}

Genet. Mol. Res. 12 (1): 738-746 (2013)

Received April 23, 2012

Accepted November 20, 2012

Published March 13, 2013

DOI http://dx.doi.org/10.4238/2013.March.13.2

\begin{abstract}
Osteoarthritis is the most common form of arthritis among elderly adults. Herein, we performed protein-protein interaction (PPI) and miRNA network analysis to evaluate the global correlation between miRNA regulation and the PPI network in human osteoarthritis. Our results showed that desmoplakin (DSP), cystatin A (CSTA), calmodulin 1, tyrosine kinase endothelial, insulin-like growth factor 1 (IGF-1), IGF-binding protein 7 (IGFBP7), syndecan 1 (SDC1), ephrin type-A receptor 4, and PDZ and LIM domain protein 1 were associated with osteoarthritis. Among these proteins, DSP and CSTA interaction and IGF-1, IGFBP7 and SDC1 interaction were observed in our PPI network. Furthermore, these potential target proteins were also linked with individual miRNA in the network. Our findings shed light on the PPIs and mechanisms by which miRNA may regulate the protein interaction network in osteoarthritis, which might provide theoretical support for further studies aimed at discovering new therapeutic strategies.
\end{abstract}

Key words: PPI network; MicroRNA network; Osteoarthritis; Protein-protein interaction 


\section{INTRODUCTION}

Osteoarthritis (OA) is the most prevalent form of arthritis in the elderly population. It features a progressive loss of articular cartilage accompanied by new bone formation and synovial proliferation that may culminate in pain, loss of joint function and disability (Rousseau and Delmas, 2007; Luyten et al., 2009). The pathologic progression of OA is generally divided into 3 stages. Stage I is characterized by the proteolytic breakdown of cartilage matrix, which results from the action of proteolytic enzymes that degrade both proteoglycans (aggrecanases) and collagen (collagenases). Native collagen could be cleaved by matrix metalloproteinase (MMP)-1, MMP-8 and MMP-13. Two aggrecanases, a disintegrin and metalloproteinase with thrombospondin motifs (ADAMTS)-4 and ADAMTS-5, appear to be the major enzymes responsible for cartilage degradation in OA (Sarzi-Puttini et al., 2005; Hardingham, 2008). Stage II is characterized by fibrillation and erosion of cartilage surface, which is accompanied by the release of breakdown products into the synovial fluid that can promote synovial inflammation, the hallmark of stage III (Martel-Pelletier, 2004). Synovial inflammation begins when synovial cells ingest a breakdown product through phagocytosis and produce proteases and proinflammatory cytokines, such as interleukin (IL)-1 $1 \beta$ and tumor necrosis factor (TNF)- $\alpha$. Both IL- $1 \beta$ and TNF- $\alpha$ exert comparable catabolic effects on chondrocyte metabolism, decreasing proteoglycan collagen synthesis and increasing aggrecan release via the induction of degradative proteases. IL-1 $\beta$ and TNF- $\alpha$ also induce chondrocytes and synovial cells to produce other inflammatory mediators, such as IL-8, IL-6, nitric oxide, and prostaglandin E2. The actions of both cytokines are in part mediated by activation of the transcription factor (TF) nuclear factor- $\kappa \mathrm{B}$, which further increases their own expression and that of other catabolic proteins such as inducible nitric oxide synthase and cyclooxygenase-2, thus creating an autocatalytic cascade that promotes self-destruction of articular cartilage (Abramson and Attur, 2009).

Network modeling of protein-protein interactions (PPIs) and miRNA-gene interactions provides a relatively new context to study disease and identify disease-related targets (Liu et al., 2011). The miRNAs represent a class of small (typically 22 nucleotides in length) non-coding RNAs that can degrade their target mRNAs or block their translation (Wu and Song, 2011). The miRNA-gene interactions could uncover disease-related regulation mechanisms. Therefore, we used the PPI and miRNA network to identify mechanisms of OA pathogenesis through evaluation of identified target genes, OA-associated genes and related gene ontology (GO) terms.

\section{MATERIAL AND METHODS}

\section{Microarray analysis}

The transcription profile of GSE19060 was obtained from the National Center for Biotechnology Information Gene Expression Omnibus (NCBI GEO) database (http://www. ncbi.nlm.nih.gov/geo/), which is based on the Affymetrix Human Genome U133A Array. Five OA meniscal cells and 3 normal controls were used. The limma method (Smyth, 2004) was employed to identify differentially expressed genes (DEGs). The original expression datasets from all conditions were extracted into expression estimates, and then used to construct the linear model. The DEGs with fold-change $>2$ and $\mathrm{P}<0.05$ were selected. 


\section{PPI network}

The Human Protein Reference Database (HPRD; Keshava Prasad et al., 2009) is a protein database accessible through the Internet. The Biological General Repository for Interaction Datasets (BioGRID; Stark et al., 2011) is a curated biological database of proteinprotein and genetic interactions. A total of 326,119 unique human PPI pairs were collected from the 2 databases and were then analyzed to match the interaction between 2 DEGs. To demonstrate the potential PPI relationship, Pearson's correlation coefficient (PCC) was calculated for all pairwise comparisons of gene-expression values between TFs and the DEGs. The PPI relationships in which the absolute PCCs were larger than 0.6 were considered significant. Then, the interactions in which the PCCs of the 2 DEGs were larger than 0.6 were selected as candidate relationships to construct the PPI network.

\section{GO-enrichment analysis}

The Database for Annotation, Visualization and Integrated Discovery (DAVID; Huang et al., 2009), a high-throughput and integrated data-mining environment, was used to analyze gene lists derived from high-throughput genomic experiments. We identified over-represented GO terms in biological process with false-discovery rate (FDR) $<0.1$.

\section{miRNA network}

The miRNAs were integrated to disease datasets and target gene datasets to determine the relationship between the disease and genes. The associations of miRNA and disease from the literature containing 444 miRNA genes, 259 diseases, 1149 publications, and 2886 miRNAdisease associations were manually retrieved from the Human MicroRNA Disease Database (HMDD; Lu et al., 2008). In addition, relationships of 349 miRNA and 163 diseases were collected form miR2Disease (Jiang et al., 2009), a comprehensive resource of miRNA deregulation in various human diseases. Five thousand and thirty-six relationships were collected after merging the 2 datasets. We also screened experimentally verified or predicted miRNA target genes from the integration of starbase (Yang et al., 2011), miR2Disease, miRecords (Xiao et al., 2009), and TarBase (Papadopoulos et al., 2009) databases and obtained 211,464 associations between miRNA and genes. The OA-related miRNAs were selected and mapped to the target genes. Finally, 137 miRNA and 14 DEGs were used to construct the miRNA network.

\section{RESULTS}

\section{PPI network construction for $\mathrm{OA}$}

The microarray dataset GSE19060 was obtained from GEO. Meniscus and articular cartilage specimens were collected from OA patients who underwent joint replacement surgery and from osteosarcoma patients who underwent lower limb amputation surgery, with the approval of the authors' Institutional Review Board. The need for informed consent was waived since the menisci and articular cartilage were surgical waste of routine joint replacement surgery and lower limb amputation surgery, and there was no private patient information 
collected. Specimens were transported to the laboratory from the operation room at our hospital in sterile tissue culture medium (Sun et al., 2010). A total of 98 DEGs with the fold-change $>2$ and 0.05 were selected in the microarray analysis. To determine the PPI relationships, the PCCs larger than 0.6 were chosen as the threshold. Finally, 17 PPI relationships including 11 DEGs and their 16 related target genes were selected. By integrating the above PPI relationships, a PPI network of OA was built (Figure 1). In this network, oxytocin receptor linked 3 target genes to form a sub-network, which suggested that it might play an important role in OA. Cystatin A (CSTA) and desmoplakin (DSP) were DEGs (Figure 1) with PCC $=0.805$ (data not shown in the graph).

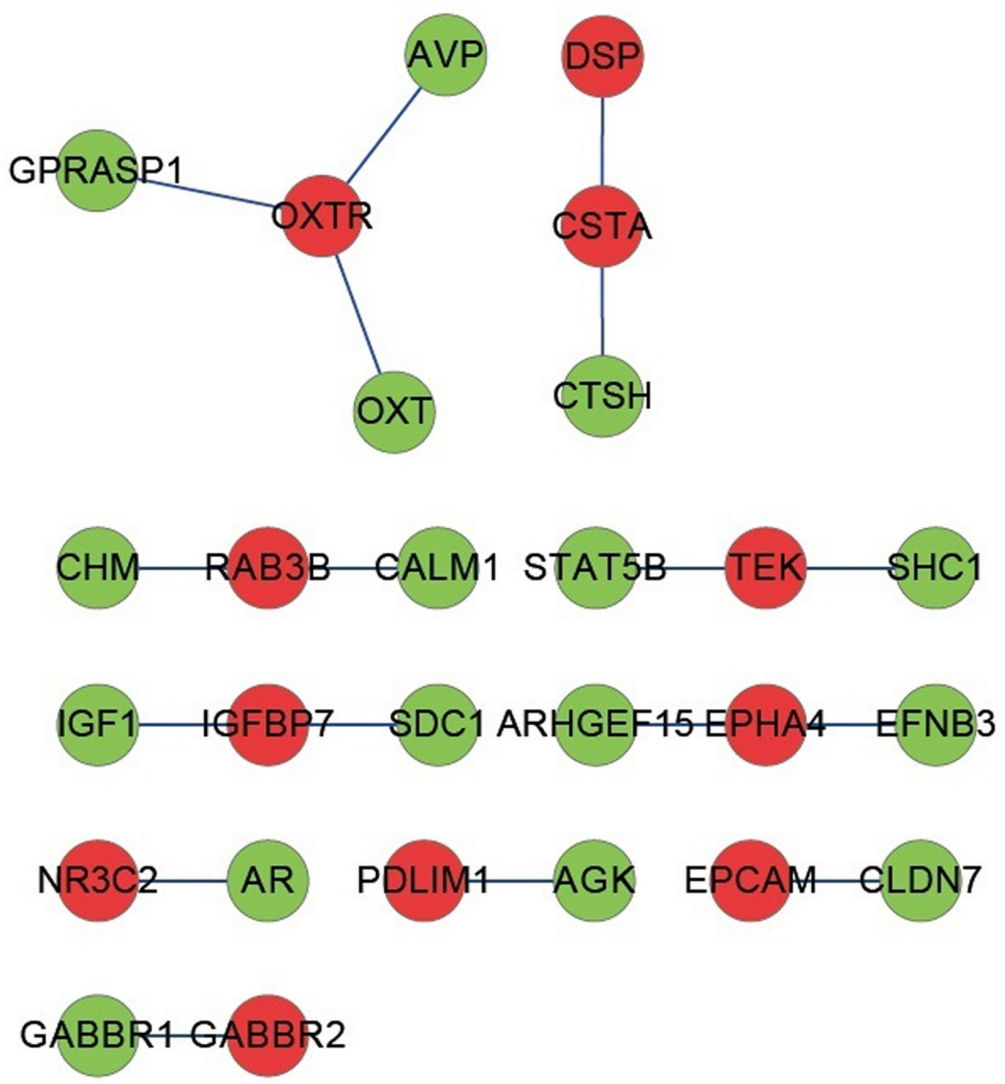

Figure 1. Protein-protein interaction network of osteoarthritis. Red nodes indicate differentially expressed genes and green nodes indicate related target genes. These nodes are connected by blue edges.

\section{GO analysis of the PPI network in OA}

Several GO terms were enriched among the 27 genes in the PPI network with the FDR $<0.1$, including response to hormone stimulus, endogenous stimulus, parental behavior, and maternal behavior (Table 1). 
Table 1. Gene ontology (GO)-enrichment analysis of biological processes.

\begin{tabular}{llccc}
\hline Category & Term & Count & P & FDR \\
\hline BP & GO 0009725 response to hormone stimulus & 7 & $3.40 \mathrm{E}-05$ & 0.051666 \\
BP & GO 0009719 response to endogenous stimulus & 7 & $5.91 \mathrm{E}-05$ & 0.089622 \\
BP & GO 0060746 parental behavior & 3 & $6.30 \mathrm{E}-05$ & 0.095609 \\
BP & GO 0042711 maternal behavior & 3 & $6.30 \mathrm{E}-05$ & 0.095609 \\
\hline
\end{tabular}

$\mathrm{BP}=$ biological process; $\mathrm{FDR}=$ false-discovery rate.

\section{miRNA network construction}

Using the miRNA-disease data collected from the HMDD and miR2 Disease database, we matched the genes in the PPI network to the miRNA-target relationships. The OArelated miRNAs were selected and mapped to other diseases. One hundred and thirty-seven miRNA and 14 genes were used to construct the network (Figure 2).

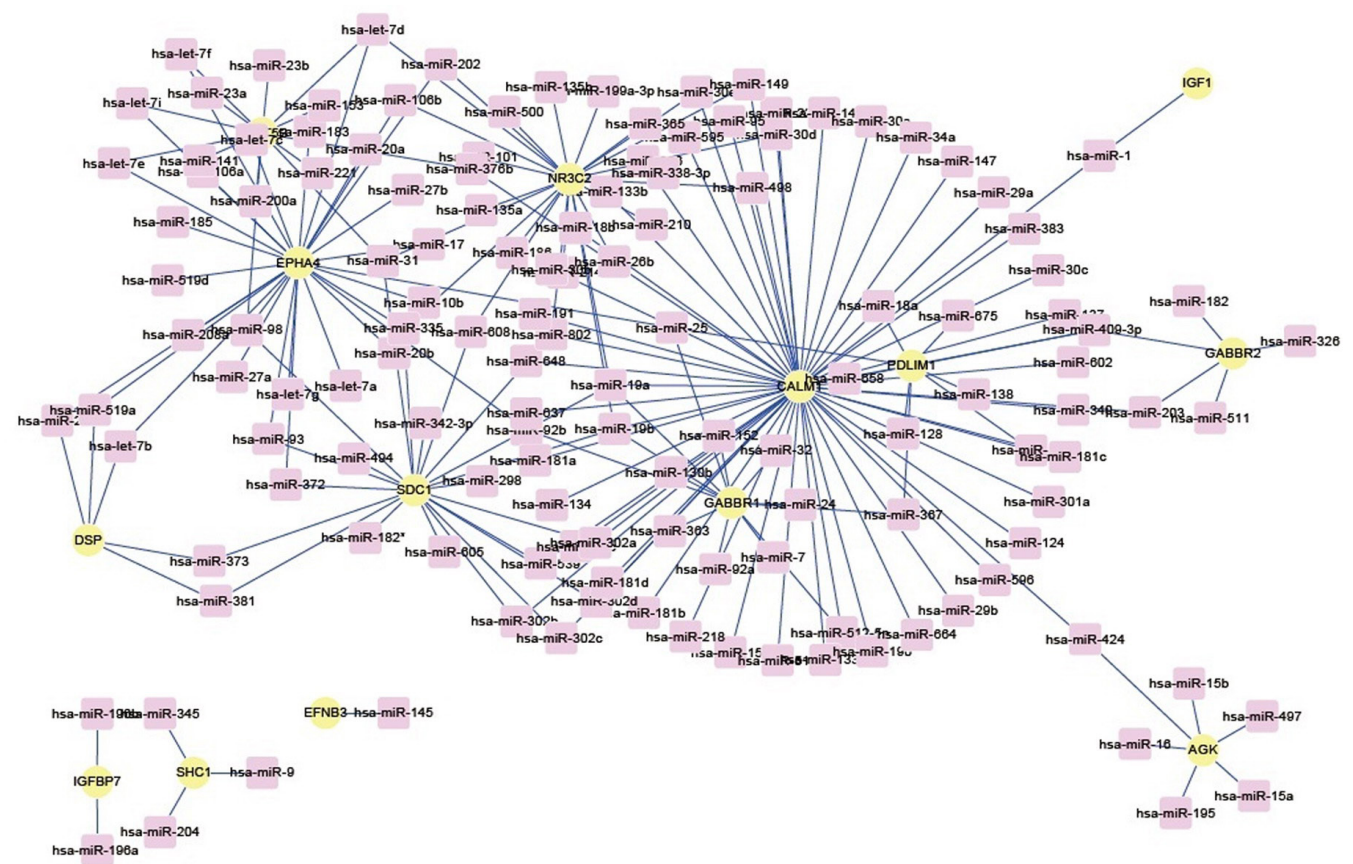

Figure 2. miRNA network of osteoarthritis. Yellow nodes indicate genes in the protein-protein interaction network and pink nodes indicate miRNA. These nodes are connected by blue edges.

\section{Overlap between the PPI and miRNA networks}

To further detect the relationships between the miRNA and PPI networks, we merged these networks to construct a new network and then selected the relationships with node de- 
grees $\geq 2$ (Figure 3 ). Syndecan 1 (SDC1), calmodulin 1 (CALM1), nuclear receptor subfamily 3 , group $\mathrm{C}$, member 2 (NR3C2), and ephrin type-A receptor 4 (EPHA4) met these criteria and were regulated by different miRNAs. hsa-miR-19b and hsa-miR-19a were responsible for SDC1, CALM1, NR3C2, and gamma-aminobutyric acid B receptor, 1 regulation in OA.

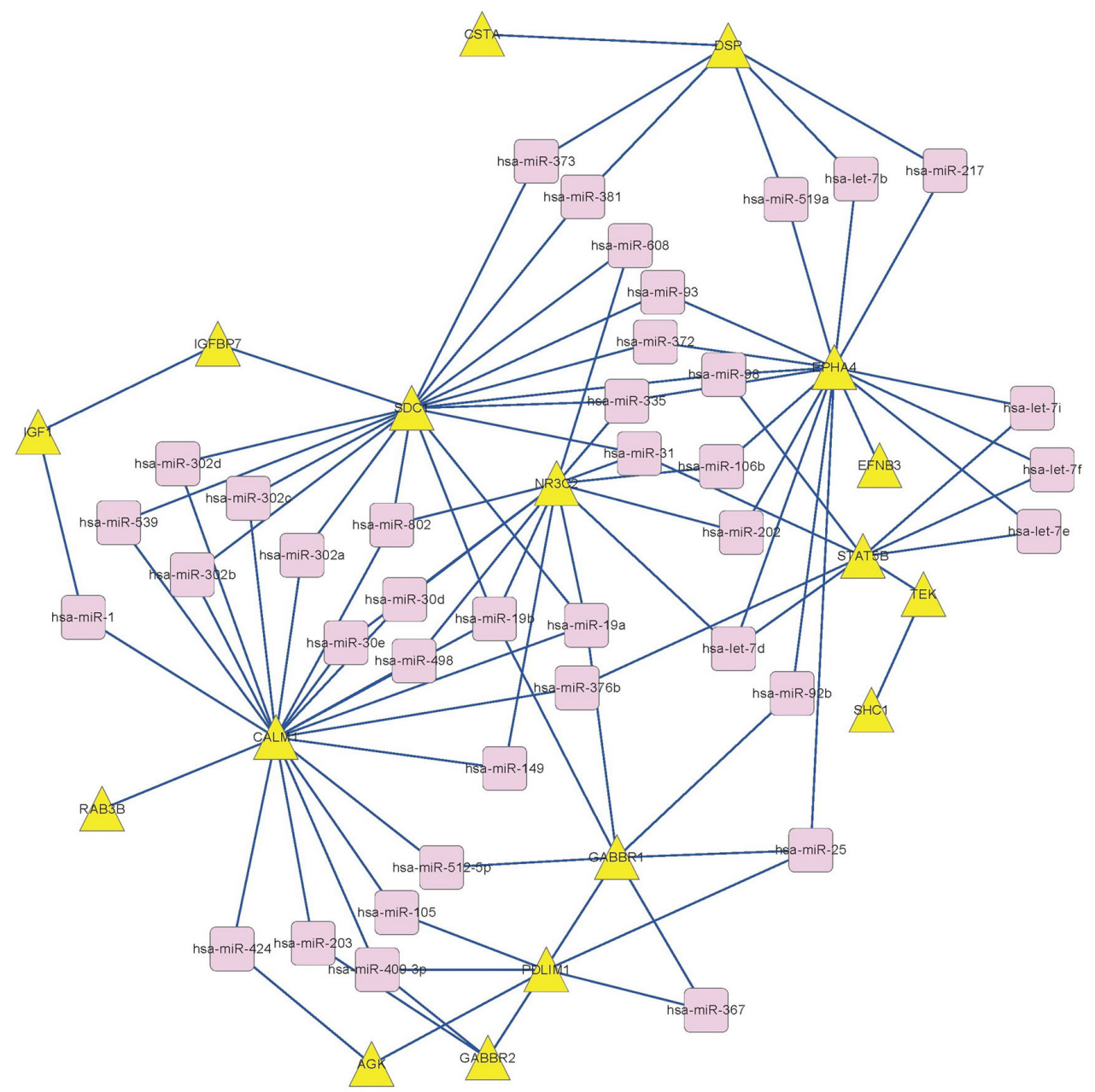

Figure 3. Overlap between protein-protein interaction network and miRNA network with degrees $\geq 2$. Yellow nodes indicate target genes, pink nodes indicate miRNAs.

\section{DISCUSSION}

The results of the present study suggested that numerous proteins and corresponding microRNAs are closely related to OA. DSP, CSTA, CALM1, tyrosine kinase endothelial (TEK), insulin-like growth factor 1 (IGF-1), IGF-binding protein 7 (IGFBP7), SDC1, EPHA4, and PDZ and LIM domain protein 1 (PDLIM1) were demonstrated to be associated 
with OA based on previous experiments. Furthermore, the interaction of IGF-1 and IGFBP7 has been identified in some diseases. CALM1 and SDC1 participated in the progression of diseases mediated by miRNAs.

DSP is an obligate component of functional desmosomes that anchors intermediate filaments to desmosomal plaques. DSP was identified as a high-abundance synovial fluid protein in OA (Gobezie et al., 2007).

CSTA is a member of inhibitory cystatin families, which functions as a cysteine protease inhibitor, forming tight complexes with papain and the cathepsins $\mathrm{B}, \mathrm{H}$ and $\mathrm{L}$ and is involved in epidermal development and maintenance. Differential expression of CSTA was observed in OA, which is consistent with decreased osteoclast activity and increased bone volume in OA (Hopwood et al., 2007).

CALM1 encodes for a ubiquitous eukaryotic $\mathrm{Ca}^{2+}$-binding protein and is the principal mediator of calcium signal. The protein thus affects the chondrocyte response to mechanical load (Poulou et al., 2008). A CALM1 single nucleotide polymorphism was reported to be associated with hip OA in Japanese individuals. CALM1 was expressed in cultured chondrocytes and articular cartilage, and its expression was increased in OA. However, this result could not be replicated in a large and well-powered study of UK Caucasian women with hip OA (Valdes et al., 2007). CALM1 has highly conserved miR-1 recognition sequences in its 3'-UTR. Both gain- and loss-of-function approaches demonstrated that miR-1 negatively regulates $\mathrm{Ca}^{2+} /$ calmodulin-dependent protein kinase levels mediated by sequence-specific interactions between miR-1 and target sequences within the CALM1 3'-UTR (Ikeda et al., 2009).

The TEK receptor tyrosine kinase is expressed almost exclusively in endothelial cells in mice, rats and humans. It is also known as TIE-2, which was significantly upregulated in the OA synovial tissue lining cells, macrophages and smooth muscle cells compared to normal synovial tissue. The ligands for TIE-2 receptor are angiopoietin-1 or angiopoietin-2. Similarly, angiopoietin-1 or angiopoietin-2 increased to participate in the later stages of angiogenesis in OA (Shahrara et al., 2002).

IGF-1 is similar to insulin in function and structure and is involved in mediating growth and development. The level of IGF-1 was increased in human OA synovial fluid. In addition, IGF-1 is also produced by OA chondrocytes, and it can act both as a paracrine and autocrine modulator to stimulate matrix synthesis and inhibit matrix degradation. Chondrocytes are hyporesponsive to IGF-1 stimulation, at least in part, to an increased level of IGFBPs (Martel-Pelletier et al., 1998). The IGFBPs have a high affinity for IGF-1, and appear to modify the interaction of IGF-1 with its receptor. The IGFBP7 gene is one member of IGFBPs, which have been linked to chromosome $4 \mathrm{q}$ and expressed in OA articular cartilage chondrocytes (Kawahara et al., 2005). Although the interaction mechanism of IGF-1 and IGFBP7 was unclear, IGFBP7 has recently been identified to modulate the anti-apoptotic effects of IGF-1 in human breast cancer (Subramanian et al., 2007).

The SDC1 protein is a transmembrane (type I) heparan sulfate proteoglycan and is a member of the syndecan proteoglycan family. The SCD1 protein functions as an integral membrane protein and participates in cell proliferation, cell migration and cell-matrix interactions via its receptor for extracellular matrix proteins. SCD1 expression was upregulated in articular cartilage during early stages of OA osteoarthritic cartilage degeneration, thus suggesting that this molecule is involved in the attempted repair of cartilage fibrillations (Barre et al., 2000; Salminen-Mankonen et al., 2005). ITF2357 is a recently identified histone deacetylase 
inhibitor of the hydroxamate family. It could inhibit several signaling pathways, such as SDC, which was downregulated by 70 and $85 \%$ in the presence of ITF2357 treatment, and demonstrated to be involved in myeloma cell growth and survival. This function was associated with hsa-miR-19a and hsa-miR-19b (Todoerti et al., 2010).

EPHA4 belongs to the ephrin receptor subfamily of the protein-tyrosine kinase family. EPH and EPH-related receptors have been implicated in mediating developmental events, particularly in the nervous system. Recently, these molecules have been implicated in the pathogenesis of OA. EPHA4 mRNA was demonstrated in activated granulocytes that participated in inflammatory processes (Ivanov and Romanovsky, 2006).

PDLIM1 belongs to the Enigma family and contains 1 PDZ and 1 LIM domain. It colocalizes with $\alpha$-actinin and actin stress fibers in different cell types and may modulate the function of $\alpha$-actinin 1. PDLIM1 expression was found to be significantly downregulated in chondrocytes, which may interfere with cytoskeletal arrangements and affect associated signaling pathways. This protein might be relevant for the pathogenesis of OA (Joos et al., 2008).

In conclusion, the PPI network and miRNA analysis is an effective avenue to screen target proteins and corresponding miRNAs to further delineate the pathobiological mechanism of OA. Further experiments are still indispensable to confirm this conclusion.

\section{REFERENCES}

Abramson SB and Attur M (2009). Developments in the scientific understanding of osteoarthritis. Arthritis Res. Ther. 11: 227.

Barre PE, Redini F, Boumediene K, Vielpeau C, et al. (2000). Semiquantitative reverse transcription-polymerase chain reaction analysis of syndecan-1 and -4 messages in cartilage and cultured chondrocytes from osteoarthritic joints. Osteoarthritis Cartilage 8: 34-43.

Gobezie R, Kho A, Krastins B, Sarracino DA, et al. (2007). High abundance synovial fluid proteome: distinct profiles in health and osteoarthritis. Arthritis Res. Ther. 9: R36.

Hardingham T (2008). Extracellular matrix and pathogenic mechanisms in osteoarthritis. Curr. Rheumatol. Rep. 10: 30-36.

Hopwood B, Tsykin A, Findlay DM and Fazzalari NL (2007). Microarray gene expression profiling of osteoarthritic bone suggests altered bone remodelling, WNT and transforming growth factor-beta/bone morphogenic protein signalling. Arthritis Res. Ther. 9: R100.

Huang dW, Sherman BT and Lempicki RA (2009). Systematic and integrative analysis of large gene lists using DAVID bioinformatics resources. Nat. Protoc. 4: 44-57.

Ikeda S, He A, Kong SW, Lu J, et al. (2009). MicroRNA-1 negatively regulates expression of the hypertrophy-associated calmodulin and Mef2a genes. Mol. Cell Biol. 29: 2193-2204.

Ivanov AI and Romanovsky AA (2006). Putative dual role of ephrin-Eph receptor interactions in inflammation. IUBMB Life 58: 389-394.

Jiang Q, Wang Y, Hao Y, Juan L, et al. (2009). miR2Disease: a manually curated database for microRNA deregulation in human disease. Nucleic Acids Res. 37: D98-104.

Joos H, Albrecht W, Laufer S, Reichel H, et al. (2008). IL-1beta regulates FHL2 and other cytoskeleton-related genes in human chondrocytes. Mol. Med. 14: 150-159.

Kawahara C, Forster T, Chapman K, Carr A, et al. (2005). Genetic association analysis of the IGFBP7, ADAMTS3, and IL8 genes as the potential osteoarthritis susceptibility that maps to chromosome 4q. Ann. Rheum. Dis. 64: 474-476.

Keshava Prasad TS, Goel R, Kandasamy K, Keerthikumar S, et al. (2009). Human Protein Reference Database - 2009 update. Nucleic Acids Res. 37: D767-D772.

Liu Y, Patel S, Nibbe R, Maxwell S, et al. (2011). Systems biology analyses of gene expression and genome wide association study data in obstructive sleep apnea. Pac. Symp. Biocomput. 14-25.

Lu M, Zhang Q, Deng M, Miao J, et al. (2008). An analysis of human microRNA and disease associations. PLoS One 3: e3420.

Luyten FP, Tylzanowski P and Lories RJ (2009). Wnt signaling and osteoarthritis. Bone 44: 522-527. 
Martel-Pelletier J (2004). Pathophysiology of osteoarthritis. Osteoarthritis. Cartilage. 12 (Suppl A): S31-S33.

Martel-Pelletier J, Di Battista JA, Lajeunesse D and Pelletier JP (1998). IGF/IGFBP axis in cartilage and bone in osteoarthritis pathogenesis. Inflamm. Res. 47: 90-100.

Papadopoulos GL, Reczko M, Simossis VA, Sethupathy P, et al. (2009). The database of experimentally supported targets: a functional update of TarBase. Nucleic Acids Res. 37: D155-D158.

Poulou M, Kaliakatsos M, Tsezou A, Kanavakis E, et al. (2008). Association of the CALM1 core promoter polymorphism with knee osteoarthritis in patients of Greek origin. Genet. Test. 12: 263-265.

Rousseau JC and Delmas PD (2007). Biological markers in osteoarthritis. Nat. Clin. Pract. Rheumatol. 3: 346-356.

Salminen-Mankonen H, Saamanen AM, Jalkanen M, Vuorio E, et al. (2005). Syndecan-1 expression is upregulated in degenerating articular cartilage in a transgenic mouse model for osteoarthritis. Scand. J. Rheumatol. 34: 469-474.

Sarzi-Puttini P, Cimmino MA, Scarpa R, Caporali R, et al. (2005). Osteoarthritis: an overview of the disease and its treatment strategies. Semin. Arthritis Rheum. 35: 1-10.

Shahrara S, Volin MV, Connors MA, Haines GK, et al. (2002). Differential expression of the angiogenic Tie receptor family in arthritic and normal synovial tissue. Arthritis Res. 4: 201-208.

Smyth GK (2004). Linear models and empirical bayes methods for assessing differential expression in microarray experiments. Stat. Appl. Genet Mol. Biol. 3: Article3.

Stark C, Breitkreutz BJ, Chatr-Aryamontri A, Boucher L, et al. (2011). The BioGRID Interaction Database: 2011 update. Nucleic Acids Res. 39: D698-D704.

Subramanian A, Sharma AK, Banerjee D, Jiang WG, et al. (2007). Evidence for a tumour suppressive function of IGF1binding proteins in human breast cancer. Anticancer Res. 27: 3513-3518.

Sun Y, Mauerhan DR, Honeycutt PR, Kneisl JS, et al. (2010). Analysis of meniscal degeneration and meniscal gene expression. BMC Musculoskelet. Disord. 11: 19.

Todoerti K, Barbui V, Pedrini O, Lionetti M, et al. (2010). Pleiotropic anti-myeloma activity of ITF2357: inhibition of interleukin-6 receptor signaling and repression of miR-19a and miR-19b. Haematologica 95: 260-269.

Valdes AM, Loughlin J, Oene MV, Chapman K, et al. (2007). Sex and ethnic differences in the association of ASPN, CALM1, COL2A1, COMP, and FRZB with genetic susceptibility to osteoarthritis of the knee. Arthritis Rheum. 56: 137-146.

$\mathrm{Wu} \mathrm{X}$ and Song Y (2011). Preferential regulation of miRNA targets by environmental chemicals in the human genome. BMC Genomics 12: 244.

Xiao F, Zuo Z, Cai G, Kang S, et al. (2009). miRecords: an integrated resource for microRNA-target interactions. Nucleic Acids Res. 37: D105-D110.

Yang JH, Li JH, Shao P, Zhou H, et al. (2011). starBase: a database for exploring microRNA-mRNA interaction maps from Argonaute CLIP-Seq and Degradome-Seq data. Nucleic Acids Res. 39: D202-D209. 